\title{
A survey of ergonomic parameters of shoppers
}

\author{
Shiva Farivar $^{1 *}$, Hassan Sadeghi Naeini ${ }^{2}$ \\ ${ }^{1}$ Iran University of Science \& Technology (IUST), Tehran, Iran \\ *Corresponding author (shivafarivar@gmail.com)
}

Purpose Shoppers are used every day to save energy and ease carrying. The lack of attention to ergonomic considerations and safety parameters can lead to injuries and related inconveniences. The purpose of this study is to assess ergonomic factors in different types of trolleys and compared to other shoppers. This will help identify proper solutions in designing an ergonomic shopper. Method In this case study, most information was obtained by interview, in depth observation and through a questionnaire. A total of 30 people with an average age of 45 volunteered to take part in the study. The findings were obtained in public places in two cities in Iran: Tehran and Mashhad. Results \& Discussion Initial results revealed that when shopping, the Iranian users' requirements were not adequately addressed. This was due to the lack of proper design and manufacturing by Iranian companies. Poor design provoked reactions and particular behaviour in users. Our results indicate dissatisfaction in the use of shopper, preventing users from buying one. Certainly, modification in shopper design with emphasis on ergonomic parameters can improve physical conditions and reduce related injuries. This study provides recommendations on how this can best be done.

Keywords: shoppers, pushing and pulling forces, handling, design factors

\section{INTRODUCTION}

Shoppers are assistance tools to save energy and ease carrying. The lack of attention to ergonomic considerations and safety parameters can lead to injuries and related inconveniences among Iranian adults and aging. This study provides recommendations in shopper design with emphasis on ergonomic parameters which improves physical conditions and reduces related injuries, hence some questions such as what forces cause to reduce injuries in arm, shoulder and low back muscles, and also what factors decreasing required forces for carrying of shopper, were answered with considering ergonomic problems in available samples.

\section{LITERATURE REVIEW}

Enid W.Y. Kwong et.al recommended that Lightness and easy storage, a pulling and pushing motion, suitability for use on stairs, and adjustable handle height were the trolley features most frequently expected by the customers. Women were more likely to be current users because they have more opportunities to shop and to purchase goods and, also, elderly people are unable to carry heavy loads, and so tend to be users of shopping trolleys [1].Therefore, other researchers recommended design factors for different types of trolleys that adds some data for ergonomic parameters of shopping trolley.

- Measure of Wheels: Drury's team showed that wheels with a $25 \mathrm{~cm}$ diameter were $16 \%$ faster than those with a $7.5 \mathrm{~cm}$ diameter. And the government of the Canadian province of Alberta suggested that carts with larger wheels are more stable, particularly when used over rough surfaces and in narrow gaps ${ }^{1}$.

- Type of wheels: Front wheel swiveling required about $10 \%$ less pushing and pulling forces ${ }^{2}$.

- Handle height: The Alberta government recommended that the handle should be at a height between the elbow and hip for pushing and between the hip and knee for pulling for optimal push and pull force capabilities ${ }^{1}$. Das et al recommended two vertical handles should be placed between 94 and $115 \mathrm{~cm}$ from the floor for a hospital meal cart. Handle height on four-wheeled carts should be at elbow height such that the force-exertion direction is close to horizontal for efficient pushing and pulling. Lee et al suggested the handle height of $150 \mathrm{~cm}$ was found in pulling and $100 \mathrm{~cm}$ for pushing with respect to the compression force at L5/S1 ${ }^{2}$. K.S. Lee found the handle height of cart should be $109 \mathrm{~cm}$ for pushing and $152 \mathrm{~cm}$ for pulling in reducing lower-back loadings ${ }^{3}$. Snook and Ciriello (1991) found that people generate higher push forces at higher handle heights, while higher pull forces are generated at lower handle heights ${ }^{4}$.

- Handle diameter: D. Welcome et al suggested hand-handle contact force is strongly dependent upon not only the grip and push forces but also the handle diameter ${ }^{5}$.

- Handle angle: Okunribido and Haslegrave (1999) recommended a handle height of 100 $\mathrm{cm}$ angled at 35 from vertical for a truck car- 
rying. Wissenden and Evans (2000) verified a $44 \%$ decrease in steering errors with an angled handle on supermarket trolleys that allowed the arms and hands to be neutral ${ }^{2}$.

- Center of mass: Idsart Kingma et al suggested that the design of Dutch twowheeled containers can be improved by moving the COM of the loaded container in the direction of the axis of the wheels and by slightly raising the height of the handles [6]. Kingma et al verified the center of load mass should be low for four-wheeled carts and should be close to the wheel axles for one- and two-wheeled hand trucks ${ }^{2}$.

- Force direction: M.P.De looze et al observed, the force direction was affected by the horizontal force level and handle height so that these were reflected in changes on the loads on the shoulder and low back in pushing and pulling wheeled carts ${ }^{7}$.

- Pushing and pulling forces: A pulling motion may also cause the cart also to run over users' feet, strike their ankles, and force them to stretch their arms behind their bodies, all of which increase the risk of pain and inju$r y^{1}$. Marco J.M et al. showed that the magnitude and direction of the exerted push force and the trunk inclination affect low back load $^{8}$. K.S. Lee et al found pushing a cart results in lesser lower-back loading than pulling and also found body weight affected the lower-back loadings more significantly in pulling $(50 \%$ increase as body weight increased from $50 \mathrm{~kg}$ to $80 \mathrm{~kg}$ ) than in pushing $(25 \% \text { increase })^{8}$. B.Schiby et al indicated that the torques at the low back and the shoulders are lower during pushing and pulling of two-wheeled waste containers compared with lifting of bags $^{9}$. Na Jin Seo showed the handle parallel to the exertion direction decreased $10 \%$ pull/push forces compared to the handle perpendicular to the exertion direction and also observed the low friction aluminum handle decreased $17 \%$ Pull/push force compared to the high friction rubber handle [10]. Martin and Chaffin found that vertical hand heights of between 50 and $90 \mathrm{~cm}$ allowed maximum pushing capability. Khaled W. Al-Eisawi et al recommended that hand forces in pushing and pulling carts are affected by cart load and handle height and also found for heavier cart loads, lower forces are applied at higher handle heights ${ }^{4}$. Marco J. M. Hoozemans suggested that in pushing the (hand) force is directed away from the body and in pulling the force is directed toward the body ${ }^{11}$.

\section{MATERIALS AND METHODS}

In this case study, the main data gathering methods were included by interview, in depth observation and questionnaire. A total of 30 people with an average age of 45 volunteered to take part in the study. The findings were obtained in public places in Tehran and Mashhad. And also the gender difference has no observably effect on analysis of information. The questionnaire is emphasized on various parameters such as way conditions for caring of shopper, keeping stable and balance in difference surfaces, the rate of energy consumption, easy of carrying, over stretching arms, the height and texture of handle, body structure.

\section{Data analysis}

With respecting of observed samples, there were no high qualities in Iranian and Chinese goods. Initial results revealed that when shopping, the Iranian users' requirements were not adequately addressed. This was due to the lack of proper design and manufacturing by Iranian companies, so that this problem provoked reactions and particular behaviour in users. The pictures depict some of above problems:

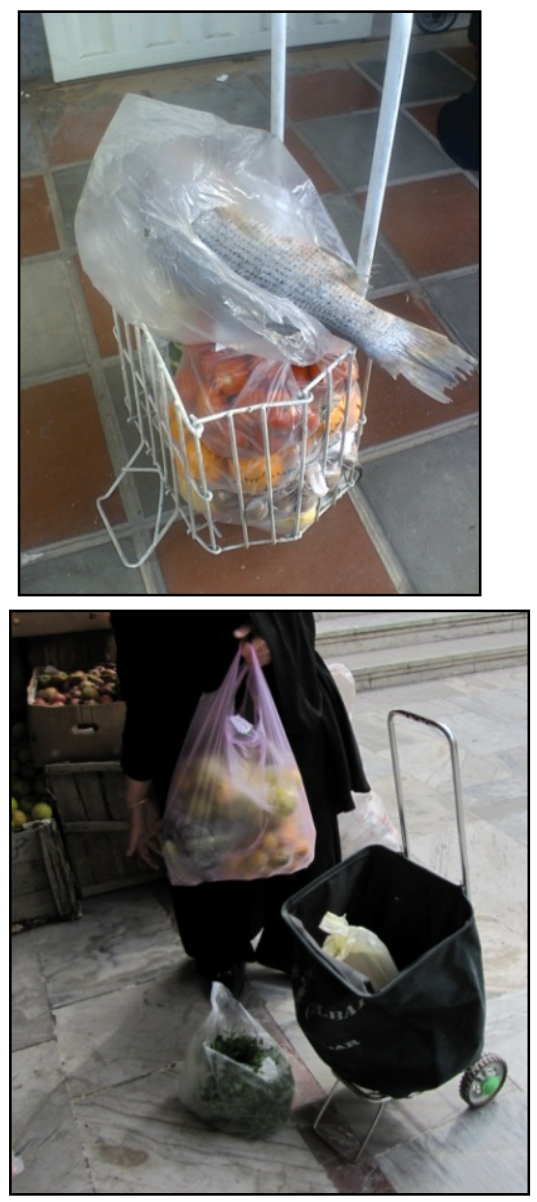



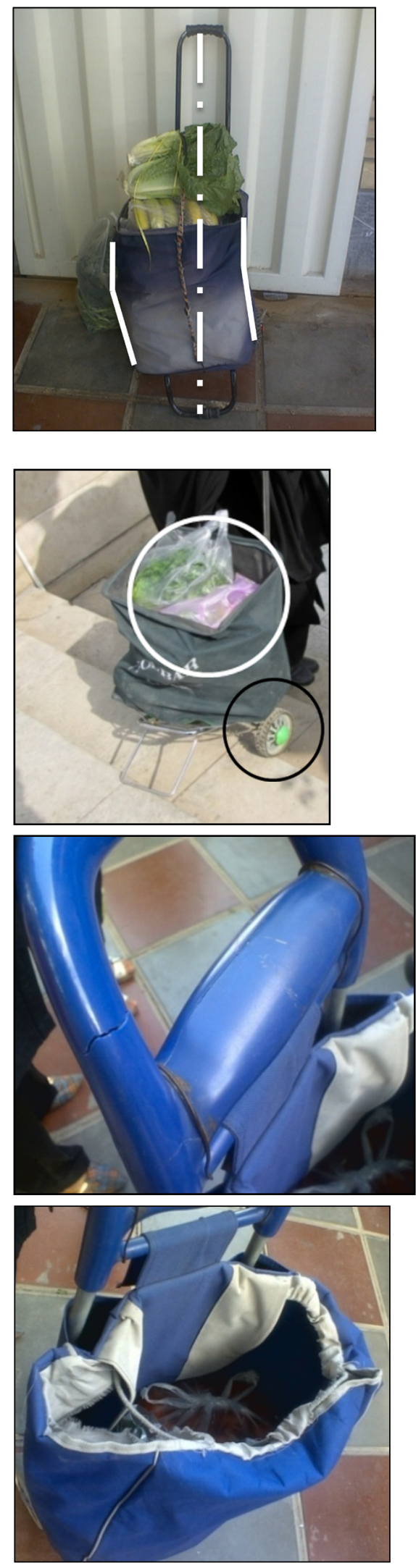
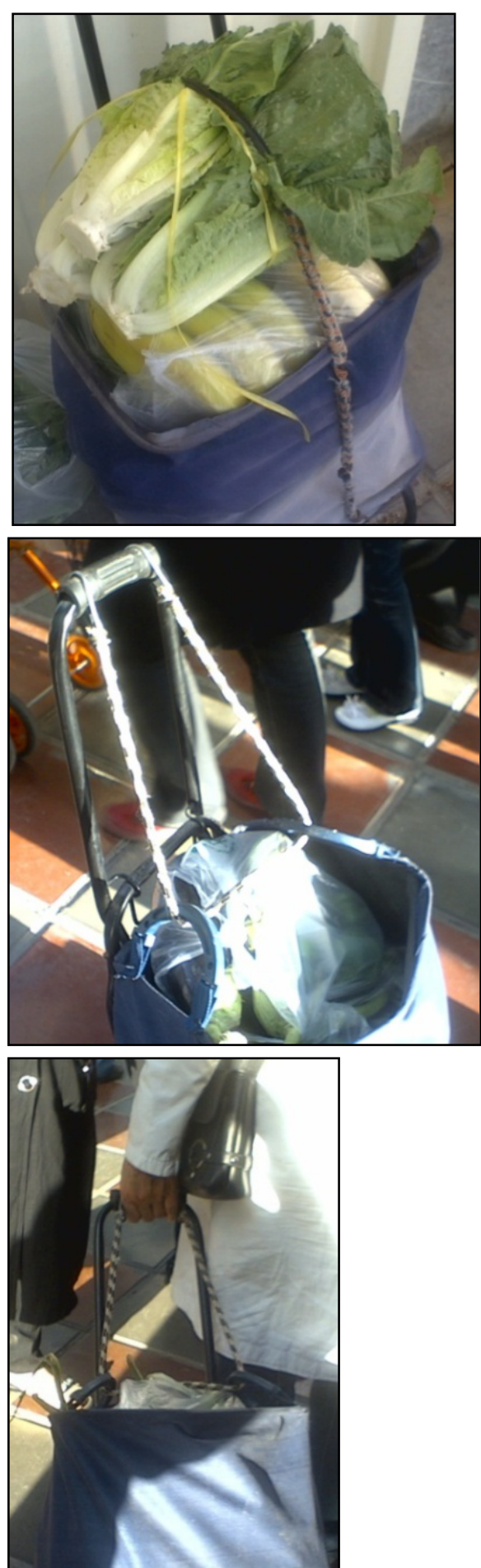

Fig. 1. Sampled cases of shopper 


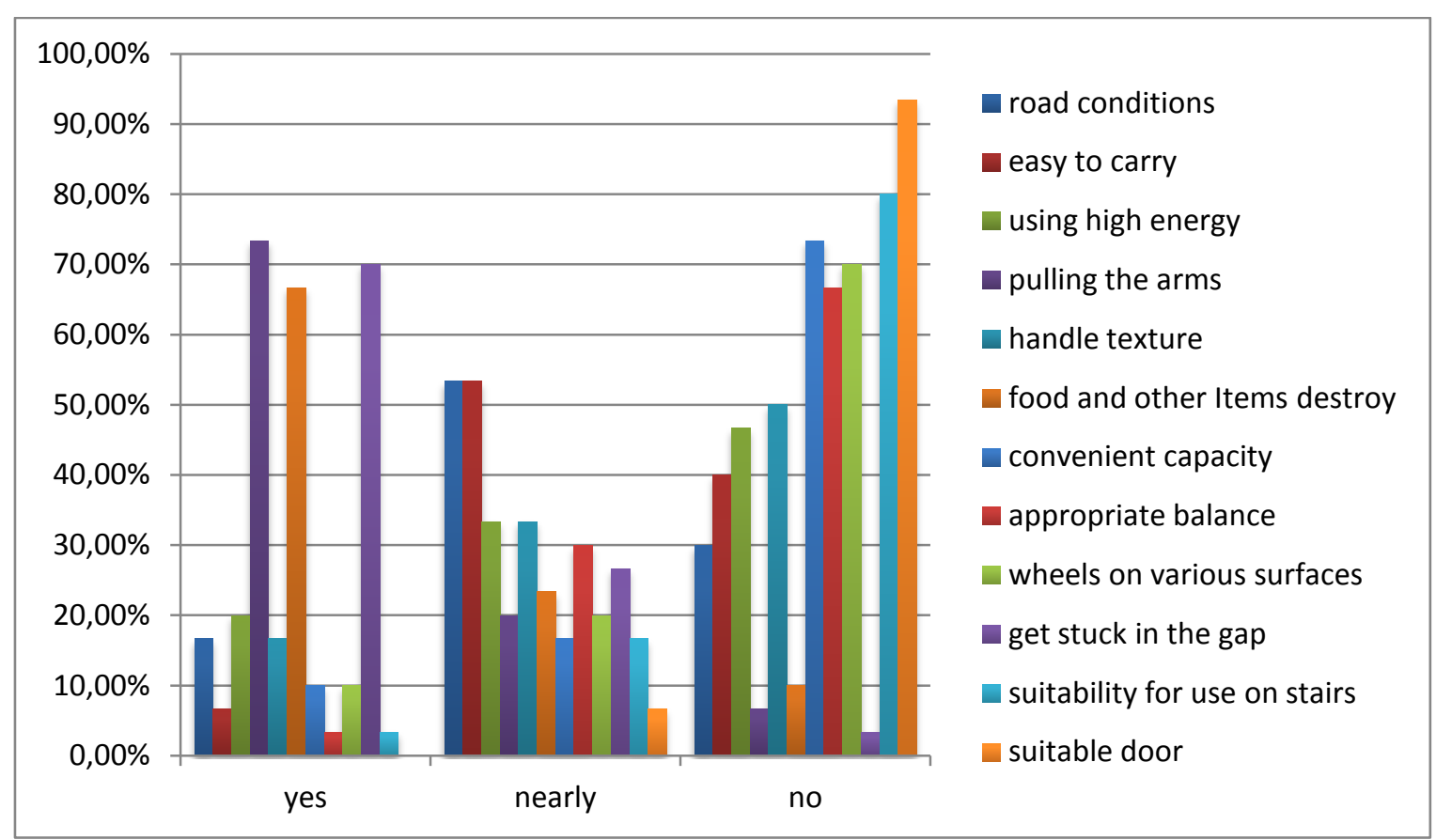

Fig. 2. The injury and dissatisfaction percentage among shoppers' users

With respecting reviews and analysis of questionnaire, it can be concluded that about $50 \%$ of people unsatisfied of carrying of shopper in different surfaces. $20 \%$ supposed that it helps in energy saving. $73.4 \%$ were injured in arm's stretching because of unadjustable handle height and unsuitable body and wheels. $66.7 \%$ complained about the unbalance of shopper. $70 \%$ supposed that is not suitable for carrying of deference spaces. Finally, $80 \%$ had some problems in coming up and down from stairs and so that had to lift it.

\section{RESULT AND DISCUSSION:}

Some problems in design of shopper can be viewed in the following:

- Wheels: transferring body vibrations by hard wheels, not easy in maneuver by non swivel wheels and getting stuck in the gap and unsuitability on stairs by small wheels.

- Handle: unsuitable angle, height and texture of handle

- Body structure: inadequate capacity and pressure exertion to items, unbalance body, unsecure loading due to unsuitable door, not easy to clean and low durable and quality.

- $\quad$ Pull and push forces: paying no attention to push force of trolley.

It doesn't meet satisfaction because manufactures didn't pay enough attention to important factors like safety and security in designing of shopper; and consequently, the rate of buying this product has significantly reduced.

\section{RECOMMENDATIONS:}

This study is the first to investigate problems and customer views on shoppers in Iran. The findings lead to modification methods with emphasis on ergonomic parameters for design of an ergonomic shopper that is acceptable to adults and aging in Tehran, Mashhad and other similar cities.

\section{Design factors:}

1. Combination of pull and push forces: The pushing force of trolley is less dangerous and safer than pulling one for body, therefore, moving forward and pushing it in straight line results in least physical stress.

2. Wheels: Rubber or polyurethane tire and spring mechanism (Anti shock system) should be used for rough places and reducing vibration in shopper, respectively. In addition to, the shopper can be capable of turning through 360 degrees by front swivel wheel.

3. Measures of Wheels: Large wheels for climbing up and down stairs and front swivel wheels should be used for lower $10 \%$ pushing and pulling forces and high maneuverability.

4. Brakes: Brake lever is used to keep fixes it in incline surfaces.

5. Body structure: Strong and stable body for keeping load balance prevents to injuries in wrist.

6. Weight: Light materials like Aluminum in its structure results in reducing physical stress.

7. Handle angle: Handle of angled at 30-35 degrees from vertical is used for easy to exerted push force.

8. Handle height: Adjustable handle $(60-95 \mathrm{~cm}$ from the floor) is used for adjusting the height of trolley handle to body. 
9. Hand-handle texture: Handle with soft foam increases more griping and reduces pressure on hand.

10. Foldable structure: The shopper can become flat for easy storage and easy to carry.

11. Type of buying: Special places are devoted for fragile and sensitive goods to prevent using several bags to gather (adjustment with type and amount of buying).

12. More facilities: Some more items are added for aging people like first aid kit, place for sitting, magnifying glass for easy reading of descriptive label (product information), safety lights for walking at night and pockets for keeping an umbrella, a walking stick, notebook, mobile, money, keys and est.
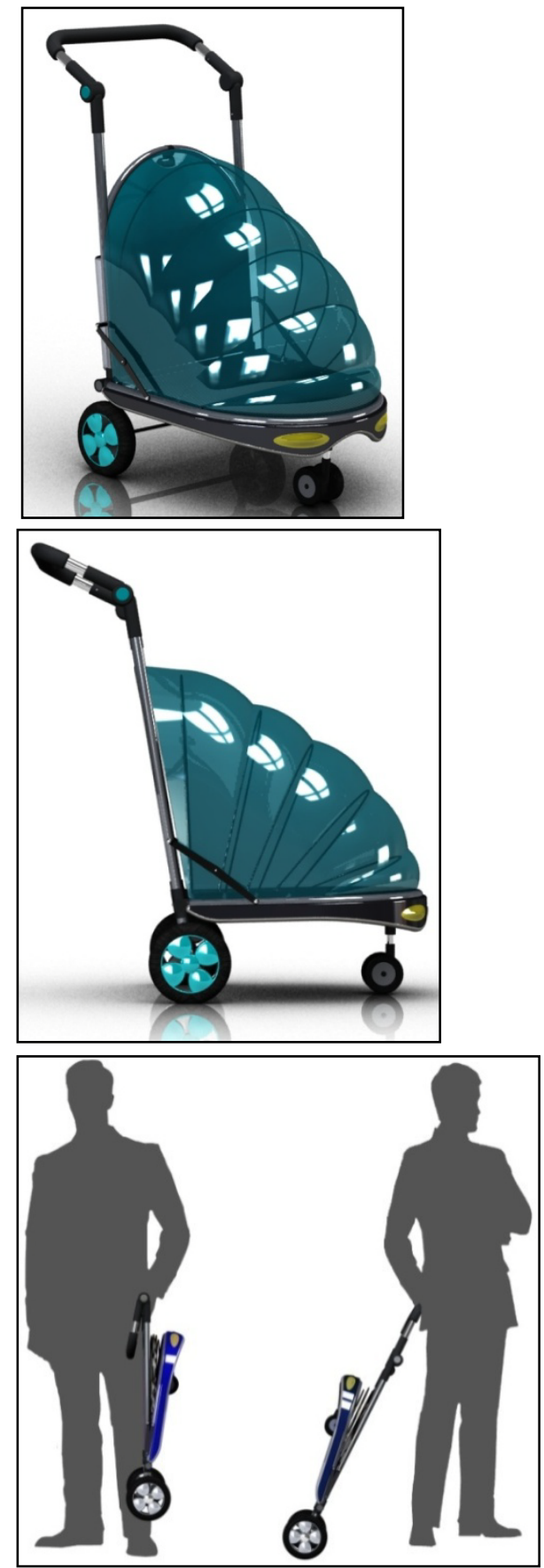

Fig.3. New concept

\section{REFERENCES}

1. Enid, W.Y., Kwong, C.K., "Views of Adults on Shopping Trolleys: Implications for the Development of a Shopping Trolley", School of Nursing \& Industrial Centre, The Hong Kong Polytechnic University, 2010.

2. Myung-Chul Junga, J.M., "Pushing and pulling carts and two-wheeled hand trucks. Industrial and Manufacturing Engineering", Pennsylvania State University, USA, 2004.

3. Lee, K.S., "Effect of handle height on lower-back loading in cart pushing and pulling", Center for Ergonomics, University of Michigan, Ann Arbor, USA, 1991.

4. Khaled, W., Al-Eisawi, C.J., "The effect of handle height and cart load on the initial hand forces in cart pushing and pulling", Department of Nuclear Engineering, Texas A\&M University, USA, 1991.

5. Welcomea, D.S.R., "An investigation on the relationship between grip, push and contact forces applied to a tool handle", Engineering \& Control Technology Branch, NIOSH, USA, 2004.

6. Idsart Kingmaa, P.P-D., "Effect of design of twowheeled containers on mechanical loading", Institute of Fundamental and Clinical Human Movement Sciences, Faculty of Human Movement Sciences, Vrije Universiteit, Amsterdam, The Netherlands, 2002.

7. Looze, M.P. de, "Force direction and phisical load in dynamic pushing and pulling", Human Movement Sciences, Vrije Universiteit, Amsterdam, The Netherlands, 2000.

8. Marco, J.M., Hoozemans, W.S., "Cart pushing: The effects of magnitude and direction of the exerted push force, and of trunk inclination on low back loading", Research Institute MOVE, Faculty of Human Movement Sciences, VU University Amsterdam, Amsterdam, Netherlands, 2006.

9. Schibye, B., Søgaard, K., Martinsen, D., Klausen, K., "Mechanical load on the low back and shoulders during pushing and puling of two-wheeled waste containers compared with", National Institute of Occupational Health, Copenhagen, Denmark, 2001.

10. Seo, N.J., Armstrong, T.J., Young, J.G., "The Effect Of Friction And Arm Posture On Max Pull / Push Force", University of Michigan, Ann Arbor, USA, 2010.

11. Hoozemans, M.J., Beek, A.J. van der, FringsDesen, M.H., Dijk, F.J. van, Woude, L.H. van der, "Pushing and pulling in relation to musculoskeletal disorders: a review of risk factors", Coronel Institute for Occupational and Environmental Health, Amsterdam, Netherlands, 1998. 